\title{
Rotura de aneurisma de la arteria hepática como causa infrecuente de dolor abdominal y hemorragia digestiva
}

\section{Ruptured hepatic artery aneurysm: an infrequent cause of abdominal pain and gastrointestinal bleeding}

\author{
Andrea Silva-Asiain, MD, ${ }^{1}$ Almudena Herrero-Domínguez, MD. ${ }^{2}$
}

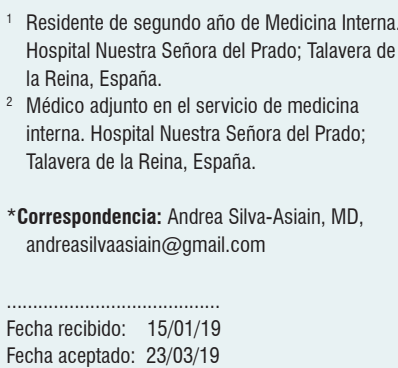

\begin{abstract}
Resumen
La hemorragia digestiva alta se define como el sangrado originado en el tracto digestivo superior, proximalmente al ángulo de Treitz, y constituye la urgencia gastroenterológica más importante. Tiene una incidencia que varía, según el área estudiada, entre 48 y 160 casos por 100000 habitantes y año. Aunque puede ser causada por numerosas etiologías, no debemos olvidar aquellas menos comunes, ya que pueden condicionar una alta mortalidad, como es el caso de la rotura de un aneurisma de la arteria hepática. A continuación, mostramos un caso representativo.
\end{abstract}

Palabras clave

Hemorragia digestiva, dolor abdominal, aneurisma visceral, aneurisma micótico.

\begin{abstract} since they can condition high mortality. We present a representative case.

Keywords

Digestive hemorrhage, abdominal pain, visceral aneurysm, fungal aneurysm.
\end{abstract}

Upper gastrointestinal bleeding is defined as bleeding originating in the upper digestive tract proximal to the Treitz angle and is the most important gastroenterological emergency. Its incidence varies, depending on the area studied, between 48 and 160 cases per 100,000 inhabitants per year. Although it can be caused by numerous etiologies, we must not forget the less common ones such as a ruptured hepatic artery aneurysm

\section{HISTORIA CLÍNICA}

Varón de 66 años con antecedentes personales de tabaquismo, hipertensión arterial y diabetes mellitus. Ingresó en enero de 2014 por una lesión ulcerada en el pie derecho, por lo cual se realizó un drenaje del absceso, con la amputación parcial del segundo dedo y, posteriormente, la amputación atípica del pie derecho.

El paciente reingresó en septiembre de 2014 por causa de una celulitis en el miembro inferior derecho y se aisló Staphylococcus aureus resistente a meticilina (SARM), así como Enterococo Faecalis. El hombre fue tratado con vancomicina, levofloxacino y linezolid con hemocultivos negativos.

Durante este ingreso, el paciente refirió epigastralgia, razón por la cual fue valorado por el servicio de digestivo y se realizaron una ecografía abdominal (hepatomegalia), una gastroscopia (lesiones ulcerosas en el duodeno, en probable relación con úlceras de estrés). Se aumentó la dosis del inhibidor de bomba de protones y una tomografía computarizada (TC) (hepatomegalia, derrame pleural bilateral [DPB] y lesiones focales en el hígado y el bazo, sugerentes de quistes simples). 
Dado el hallazgo de un DPB, el paciente fue valorado por medicina interna con sospecha de insuficiencia cardíaca. Ante ello, se realizó un ecocardiograma en el cual se evidenció una válvula aórtica calcificada y desestructurada, con estenosis moderada e insuficiencia ligera a moderada.

Un mes después, ingresó de nuevo por una sepsis secundaria a una herida infectada en el pie derecho, con osteomielitis secundaria. Se aisló Stenotrophomonas maltophila, Pseudomona aeruginosa y SARM. Así pues, se realizó la amputación infracondílea y se inició una antibioterapia con Tazocel ${ }^{\oplus}$ y linezolid.

En este nuevo ingreso, se objetivó una bacteriemia por SARM realizando un ecocardiograma transtorácico, tras lo cual se observó una progresión de la insuficiencia aórtica de grado moderada a severa, así como hipertensión pulmonar y una dilatación biauricular que no existía previamente. El paciente fue dado de alta con linezolid, pero persistió, durante este ingreso, el dolor abdominal.

\section{EVOLUCIÓN}

A las $48 \mathrm{~h}$ del alta, el paciente acudió de nuevo al servicio de urgencias, a causa de una epigastralgia persistente y sufriendo una parada cardiorrespiratoria (PCR). Tras la reanimación cardiopulmonar avanzada, el hombre se recuperó e ingresó en la unidad de vigilancia intensiva (UVI). Allí, luego de la realización de un ecocardiograma transesofágico, se diagnosticó una endocarditis sobre la válvula nativa aórtica. Por tanto, el paciente fue trasladado al centro de referencia para efectuársele una cirugía cardíaca.

Se implantó una prótesis aórtica y se inició el tratamiento con daptomicina, gentamicina y rifampicina. Poco tiempo después, el paciente fue trasladado al servicio de cardiología de nuestro centro, a fin de completar el tratamiento antibiótico.

Durante su estancia en Cardiología, comenzó con dolor abdominal y colestasis. Además, se observaron las siguientes características: una alanina-aminotransferasa (ALT) de 207 U/L (2-33); una aspartato-aminotransferasa (AST) de 74 U/L (1-31); fosfatasa alcalina (FA) de 825 U/L (35-129), y una $\gamma$-glutamiltransferasa (GGT) de $1987 \mathrm{U} / \mathrm{L}$ (7-40).

Se realizó una TC abdominal, en la cual se observó la dilatación del árbol biliar intrahepático bilateral y del colédoco proximal hipercaptante, con calibre filiforme distal. También se efectuó una ecografía abdominal que confirmó la permeabilidad de la vena porta y de sus ramas, así como de la vena mesentérica superior. Y se efectuó una gastroscopia (endoscopia digestiva alta, EDA) que resultó normal. En esta situación, el paciente fue trasladado al servicio de digestivo.

El hombre continuó con dolor epigástrico y presentó varios episodios de hemorragia digestiva en forma de melenas, vómitos «en posos de café» y rectorragia con anemización que precisó de transfusión. Así pues, se realizó una
EDA en varias ocasiones y únicamente se objetivaron 3 erosiones esofágicas y gastritis crónica. Además, se efectuó una colonoscopia sin hallazgos.

Con respecto a la exploración física, no parecía haber ningún hallazgo relevante. Sin embargo, 24 h después de la última gastroscopia normal, el paciente presentó un episodio de rectorragia y hematemesis, con choque hemorrágico secundario, por lo que fue ingresado en la unidad de cuidados intensivos (UCI).

De nuevo, se practicó una EDA urgente, en la cual se evidenció un abundante contenido hemático en el estómago y un coágulo alargado en la papila, con babeo adyacente a nivel de esta, lo que fue sugerente de hemobilia.

A partir de estos hallazgos, se realizó una angiotomografía computarizada (angio-TAC) (Figura 1), que permitió objetivar el aneurisma de una rama segmentaria de la arteria hepática derecha, con contenido fusiforme sugerente de hematoma intraluminar biliar.

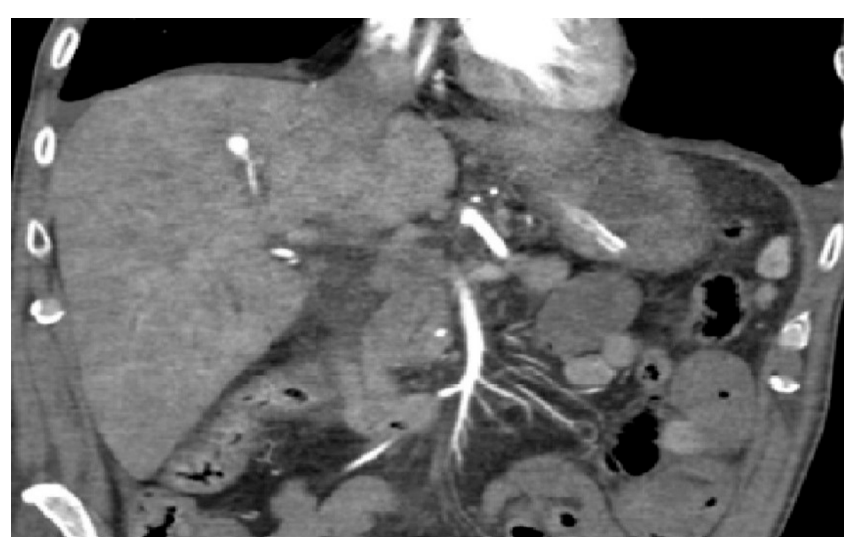

Figura 1. Aneurisma de una rama segmentaria de la arteria hepática derecha, en el segmento V.

Se procedió entonces a la embolización del aneurisma (Figura 2), con lo cual el paciente evolucionó favorablemente, sin más episodios de sangrado digestivo y con la resolución completa de la obstrucción biliar.

\section{DIAGNÓSTICO}

Hemorragia digestiva alta, causada por la rotura de un aneurisma de una rama segmentaria de la arteria hepática derecha.

\section{DISCUSIÓN}

Existen múltiples causas de dolor abdominal y entre ellas, de forma infrecuente, aparece descrito el aneurisma de la arteria abdominal. Paralelamente, al revisar la literatura, la hemobilia se halla como un desencadenante inusual de 

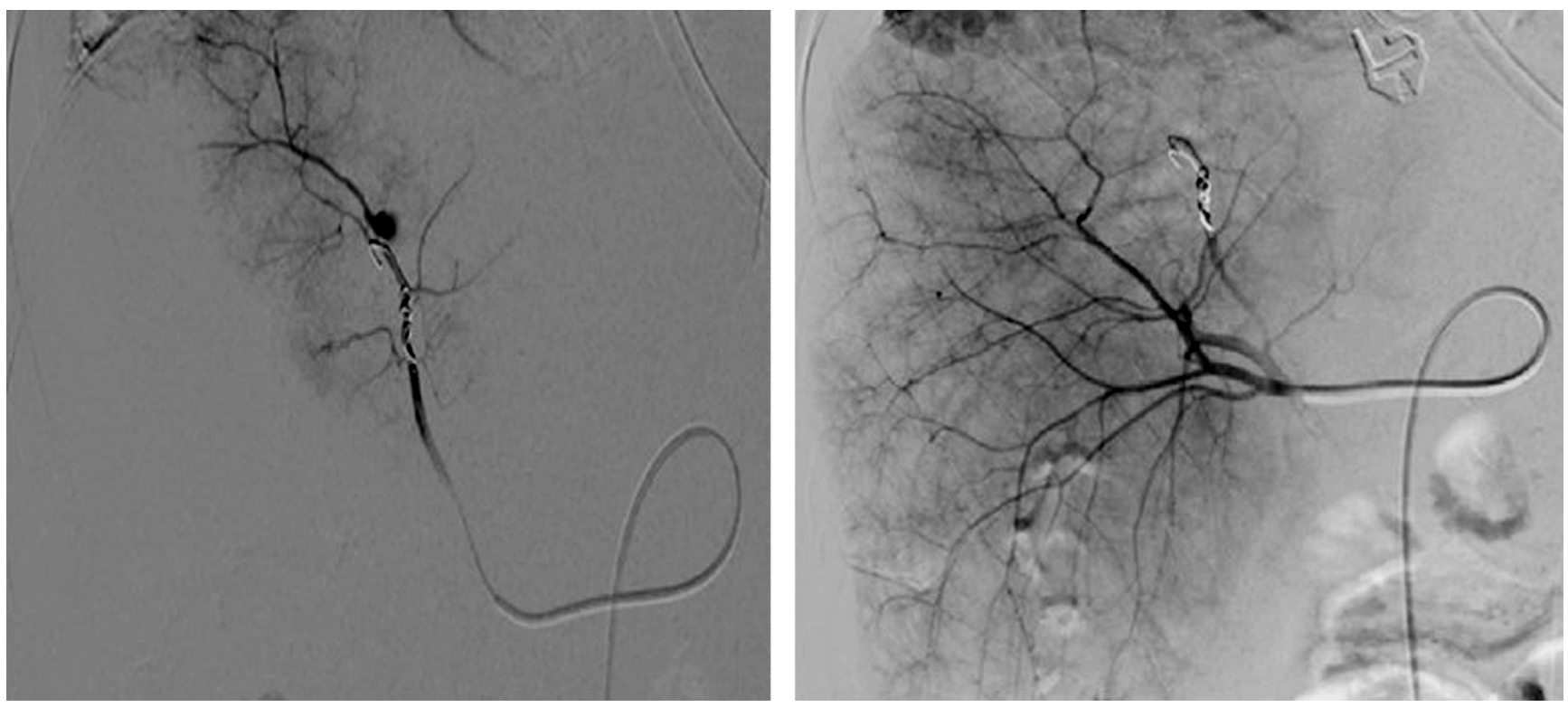

Figura 2. Arteriografía hepática y embolización del aneurisma de la arteria hepática.

hemorragia digestiva alta, al alcance del gastroscopio, y que no es causado directamente en el intestino delgado. Por tanto, se considera una causa de sangrado digestivo de origen oscuro $(1,2)$.

La clínica de la hemobilia se caracteriza por la aparición de ictericia, dolor en el hipocondrio derecho y hemorragia digestiva alta (3-5), síntomas que se presentaron en nuestro paciente. Las causas descritas son traumáticas, iatrogénicas, inflamatorias, infecciosas, neoplásicas y vasculares, tales como los aneurismas, las vasculitis y las malformaciones arteriovenosas.

En consecuencia, el diagnóstico se realiza mediante una prueba de imagen $(6,7)$, ya sea una TC, una EDA o una resonancia magnética nuclear (RMN) (8). El tratamiento de elección es la embolización selectiva, pero existen otras opciones como la inserción de un stent endovascular o el tratamiento quirúrgico $(3,9,10)$.

En nuestro caso, el origen fue un aneurisma de la arteria hepática, considerado el segundo aneurisma de arterias viscerales más frecuente, solo por detrás del esplénico (11-13). Si nos centramos específicamente en la etiología de los aneurismas de la arteria hepática, observamos que las causas son diversas e incluyen la arteriosclerosis -como entidad más frecuente-, el traumatismo hepático, la micosis (hasta un $10 \%$ ), la adicción a drogas por vía parenteral, causas iatrogénicas y las enfermedades autoinmunes, entre otras (14).
A nuestro paciente podría atribuírsele la etiología del aneurisma visceral a dos causas: la arteriosclerosis, objetivada en varias pruebas de imagen, y el aneurisma micótico, por el antecedente reciente de endocarditis con bacteriemia por SARM (causa y germen más frecuente en los aneurismas infectados).

Así, tanto la sintomatología como la exploración de los aneurismas viscerales suelen ser anodinas y, por tanto, en la mayoría de los casos, conducen a hallazgos incidentales cuando se realiza una prueba de imagen. La excepción a ello se da cuando dichos aneurismas se rompen y pueden ocasionar hemobilia si esto ocurre hacia la vía biliar. Con este panorama, el tratamiento debe ser precoz, ya que ello puede condicionar una mortalidad que oscila entre el $50 \mathrm{y}$ el $100 \%$, según las series de casos $(3,11,14)$.

\section{CONCLUSIONES}

La asociación entre la hemorragia digestiva alta y la colestasis nos debe conducir a pensar en la hemobilia y sus causas. Por otro lado, en el diagnóstico diferencial del dolor abdominal y la hemorragia digestiva alta de origen no filiado debemos incluir los aneurismas de arterias viscerales. Estos, a pesar de ser poco comunes, pueden llegar a causar la muerte del paciente si no se actúa a tiempo. 


\section{REFERENCIAS}

1. Gerson LB, Fidler JL, Cave DR, Leighton JA. ACG Clinical Guideline: Diagnosis and Management of Small Bowel Bleeding. Am J Gastroenterol. 2015;110(9):1265-1288. http://doi.org/10.1038/ajg.2015.246

2. Welch GH, Anderson JR, Leiberman DP. Aneurysms of the hepatic artery: a report of three cases. J R Coll Surg Edinb. 1986;31(2):91-94.

3. Bellmunt-Montoya S, Maeso-Lebrún J, Royo-Serrando J, Bofill-Brosa R, Allegue-Allegue N, Matas-Docampo M. Aneurismas de la arteria hepática. Descripción de cinco casos y revisión de la literatura. Angiología. 2001;53(1):33-41. http://doi.org/10.1016/S0003-3170(01)74675-5

4. Santos VM, Villaça RB, Almeida AC, Oliveira ER, Nogueira PR, Damasceno EA. Rupture of hepatic artery aneurysm associated with ischemic hepatitis. An Sist Sanit Navar. 2010;33(2):221-225.

http://doi.org/10.4321/s1137-66272010000300011

5. Galindo P, Martínez JD, Rey MH. Hemobilia secundaria a aneurisma de la arteria hepática. Rev Colomb Gastroenterol. 2017;32(2):171-173. https://doi.org/10.22516/25007440.145

6. Rencuzogullari A, Okoh AK, Akcam TA, Roach EC, Dalci $\mathrm{K}$, Ulku A. Hemobilia as a result of right hepatic artery pseudoaneurysm rupture: An unusual complication of laparoscopic cholecystectomy. Int J Surg Case Rep. 2014;5(3):142-144. http://doi.org/10.1016/j.ijscr.2014.01.005
7. Panno C, Gutiérrez SC, Echeverría R, Garbe C, Milocco M, Ianniccillo H, Valenzuela M, Cubilla A, Álvarez F, Álvarez E. Hemobilia secundaria a pseudoaneurisma de la arteria hepática. Acta Gastroenterol Latinoam. 2015;45(2):133-136.

8. Busuttil RW, Brin BJ. The diagnosis and management of visceral artery aneurysms. Surgery. 1980;88(5):619-624.

9. Beltrán F, Castillo A, Hidalgo H. Hemobilia secundaria a pseudoaneurisma roto: reporte de caso. Rev Gastroenterol Perú. 2015;35(1):89-92.

10. Jonsson K, Bjernstad A, Eriksson B. Treatment of a hepatic artery aneurysm by coil occlusion of the hepatic artery. AJR Am J Roentgenol. 1980;134(6):1245-1247. http://doi.org/10.2214/ajr.134.6.1245

11. Puppo AM, Pareja F, Muñoz J, Forastero A, Bernardos A. Aneurisma de la arteria hepática. Cir Esp. 2007;81(6):348-50. http://doi.org/10.1016/S0009-739X(07)71336-2

12. Stanley JC, Wakefield TW, Graham LM, Whitehouse WM Jr, Zelenock GB, Lindenauer SM. Clinical importance and management of splanchnic artery aneurysms. J Vasc Surg. 1986;3(5):836-840. https://doi.org/10.1016/0741-5214(86)90059-5

13. Graham JM, McCollum CH, DeBakey ME. Aneurysms of the splanchnic arteries. Am J Surg. 1980;140(6):797. http://doi.org/10.1016/0002-9610(80)90120-8

14. Cases-Baldó MJ, Guillen-Paredes MP, Moreno-Egea A, Torres-Del Río S, Aguayo-Albasini JL. Rotura aneurismática de la arteria hepática común. Cir Esp. 2010;87(1):46- 58. http://doi.org/ 10.1016/j.ciresp.2008.10.014 